\title{
A New Scintillator Tile/Fiber Preshower Detector for the CDF Central Calorimeter
}

\author{
Michele Gallinaro ${ }^{7}$ \\ on behalf of
}

\author{
A. Artikov, ${ }^{2}$ C. Bromberg, ${ }^{5}$ J. Budagov, ${ }^{2}$ K. Byrum, ${ }^{1}$ S. Chang, ${ }^{4}$ G. Chlachidze, ${ }^{2}$ K. Goulianos, ${ }^{7}$ J. Huston, ${ }^{5}$ \\ M. Iori, ${ }^{8}$ M. Kim, ${ }^{4}$ S. Kuhlmann, ${ }^{1}$ S. Lami, ${ }^{7}$ M. Lindgren, ${ }^{3}$ E. Lytken, ${ }^{3}$ R. Miller,${ }^{5}$ L. Nodulman, ${ }^{1}$ G. Pauletta, ${ }^{9}$ \\ A. Penzo, ${ }^{9}$ J. Proudfoot, ${ }^{1}$ R. Roser, ${ }^{3}$ J. Suh,${ }^{4}$ N. Turini, ${ }^{6}$ F. Ukegawa, ${ }^{10}$ R. Wagner ${ }^{1}$ \\ 1 Argonne National Laboratory, Argonne, Illinois 60439 \\ 2 Joint Institute for Nuclear Research, RU-141980 Dubna, Russia \\ 3 Fermi National Accelerator Laboratory, Batavia, Illinois 60510 \\ 4 Korean Hadron Collider Laboratory: Kyungpook National University, Taegu 702-701; Korea \\ 5 Michigan State University, East Lansing, Michigan 48824 \\ 6 Istituto Nazionale di Fisica Nucleare, University of Siena, Italy \\ 7 The Rockefeller University, New York, New York 10021 \\ 8 Istituto Nazionale di Fisica Nucleare, University of Roma, Italy \\ 9 Istituto Nazionale di Fisica Nucleare, University of Trieste/Udine, Italy \\ 10 University of Tsukuba, Tsukuba, Ibaraki 305, Japan
}

\begin{abstract}
A detector designed to measure early particle showers has been installed in front of the central CDF calorimeter at the Tevatron. This new preshower detector is based on scintillator tiles coupled to wavelength-shifting fibers read out by multianode photomultipliers and has a total of 3,072 readout channels. The replacement of the old gas detector was required due to an expected increase in instantaneous luminosity of the Tevatron collider in the next few years. Calorimeter coverage, jet energy resolution, and electron and photon identification are among the expected improvements. The final detector design, together with the $R \& D$ studies that led to the choice of scintillator and fiber, mechanical assembly, and quality control are presented. The detector was installed in the fall 2004 Tevatron shutdown and is expected to start collecting colliding beam data by the end of 2004 . First measurements indicate a light yield of 12 photoelectrons/MIP, a more than two-fold increase over the design goals.
\end{abstract}

Index Terms - scintillation detectors, optical fibers, calorimetry.

\section{INTRODUCTION}

$\mathbf{T}$ HE physics program at the Fermilab Tevatron collider will continue to explore the frontier of particle physics until the startup of the LHC experiments at CERN later in this decade. Meanwhile, in preparation for the expected large data output from the Tevatron collider program, the instantaneous luminosity increase during the second phase of Run II and a better detector performance require detector upgrades beyond the ones first planned for the start of Run II. Calorimeter performance can be improved by replacing the old preshower gas detector with a new detector based on scintillator technology. During the fall 2004 Tevatron shutdown, the front face of the CDF central calorimeter has been equipped with a new preshower detector based on scintillator tiles and read out by wavelengthshifting (WLS) fibers. Thanks to a larger signal output from the new detector and better signal to noise ratio, both electron and photon identification are expected to improve significantly.

\section{WHY A NEW DETECTOR?}

$\mathbf{T}$ HE replacement of the Central Preshower (CPR) and Central Crack (CCR) detectors with scintillator detector technology is recommended for a number of reasons:

1) The old CPR is a slow wire chamber with a relatively poor segmentation; the instantaneous luminosity has increased since the start of Run II by a factor of ten, and a further rise is expected in the next few years. This combination would lead to high detector occupancy, jeopardizing good electron and photon identification during the most crucial period of Run II, when the high-energy physics frontier can be explored with large data samples before the LHC era arrives.

2) Jet energy corrections can be improved even at the current occupancy level with an expanded detector segmentation.

3) The old gas detectors have been operating since the start of Run I and wire aging is degrading the capability of the old detector. Furthermore, the signal pulses from a gas detector are small and signal response resolution is poor, when compared to those from a scintillator detector. Both effects are of crucial importance to electron and photon identification and energy resolution improvements.

4) Electron-pion separation can also be improved using a new detector with an improved ability to detect minimum ionizing 
particles (MIPs) and separate them from the background of pions (see, for example, Ref. [1], p. 2996).

\section{EXTENDING THE PHYSICS REACH}

$\mathbf{T}$ HE CPR has already contributed to many important physics results, especially those involving photon and electron identification. The upgrade of the CDF central calorimeter is expected to greatly improve the Run II physics results and enhance the sensitivity to physics beyond the standard model. In particular, it will play an important role in soft electron tagging of $b$-jets, photon identification, and jet resolution. Jet resolution can be improved by incorporating tracking information in the jet reconstruction algorithm [2]. Improvement of jet energy resolution can be important in enhancing the reach for new physics processes, such as the Higgs boson. For the purpose of photon identification at CDF, the shower maximum detector alone cannot resolve single photons from meson decays (i.e. $\pi^{0} \rightarrow \gamma \gamma$ ) above $35 \mathrm{GeV}$, as the angular separation between the two photons is too small. Instead, the CPR uses photon conversion rates which are energy independent. Furthermore, it can be used to estimate the backgrounds to exotic physics signals that include photons. In fact, if present, new physics events are expected to appear in the high transverse momentum region where the shower maximum has no discriminatory power.

The thin gas layers of the old CCR detectors have not been used efficiently due to several reasons, including low signal response. However, the capability of tagging high energy electrons and photons in the crack region in events which may contain new physics is of paramount importance. For example, if supersymmetry manifests itself, as some suggest, in events with photons and missing transverse energy [3], a better calorimeter coverage is highly desirable.

\section{DETECTOR DESIGN}

$\mathbf{T}$ HE upgrade of the CDF central calorimeter [4] includes the replacement of the CPR and CCR detectors, which sample the early particle showers in front of the central calorimeter (Fig. 1). The slow gas detectors, which have been in operation since the start of Run I, have just been replaced during the Tevatron shutdown in the fall of 2004, with a faster scintillator version and better segmentation.

The new CPR detector is made of $20-\mathrm{mm}$ thick scintillator tiles (Fig. 2) read out through a 1-mm diameter WLS fiber embedded into a groove carved on the surface of each tile. The groove cut inside the scintillator's surface has a 2-loop spiral design with a cross-sectional keyhole shape in order to maximize light collection. After exiting the tile, each WLS fiber is spliced to a clear fiber, which terminates in a plastic connector at the module's edge. Optical cables, approximately $5 \mathrm{~m}$ long, then transport the light to a 16-channel R5900 Hamamatsu Photo-Multiplier Tube (PMT) located in the back of the calorimeter wedge. Each detector channel (i.e. one tile) is read out by one PMT pixel, for a total of 2,592 (480) readout channels in the CPR (CCR) detector.

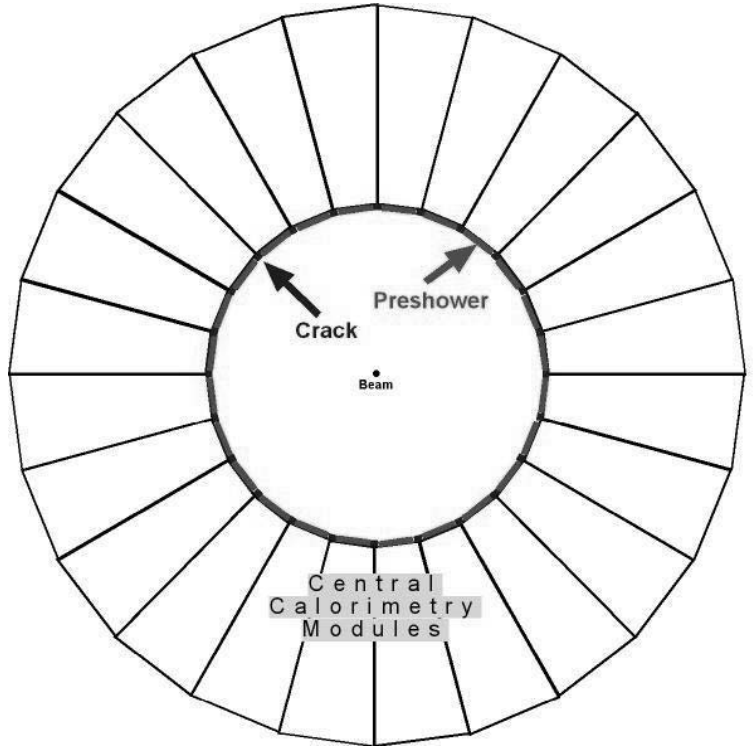

Fig. 1. The central calorimetry wedges and the location of the Preshower and Crack detectors.

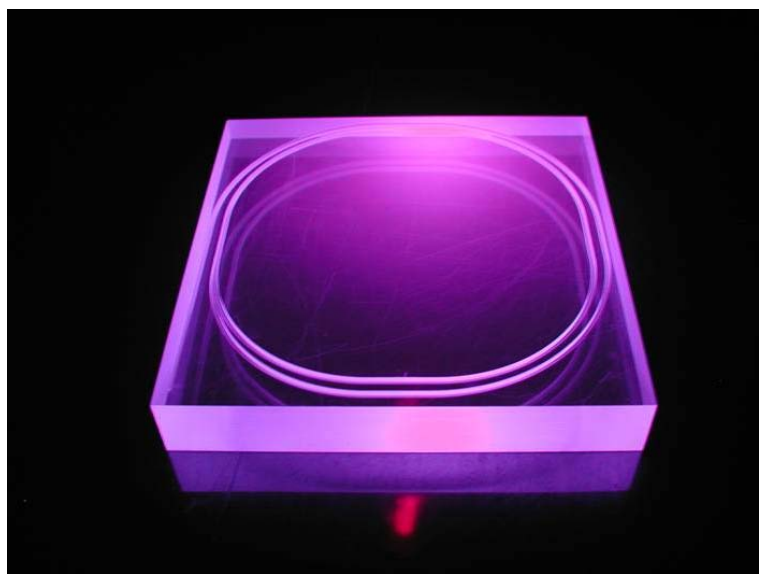

Fig. 2. CPR tile carved with a two-loop spiral groove path.

The CCR detector is replaced by a similar detector read out through the same technique. The CCR is located behind a 10 radiation length tungsten bar, which limits the scintillator thickness to $5 \mathrm{~mm}$ and covers the uninstrumented regions, also called "cracks", in the azimuthal angle $\phi$ present between the calorimeter wedges. Ten tiles, approximately $5 \mathrm{~cm}$ wide, cover each $\phi$-crack with the same $\eta$-segmentation as that of the central calorimeter of 10 towers per wedge. One WLS fiber is embedded into a straight groove in each tile.

Both the CPR and the CCR detectors use the solenoid coil and the tracking material as a radiator. The entire detector consists of a total of 48 CPR (and 48 CCR) modules, with each module covering the front face of one calorimeter wedge. A set of 54 tiles forms one CPR module (Fig. 3) and is assembled in an aluminum shell, sealed to be light-tight. The old CPR detector had a poorer segmentation with only $16 \times 2$ towers for each calorimeter wedge [6]. In the new CPR modules, a 
continuous array of $3 \times 18$ tiles $\left(12.5 \times 12.5 \mathrm{~cm}^{2}\right.$ each $)$ spans the face of one calorimeter wedge. The front-end electronics uses the same readout modules previously employed by the old detector, with only minor modifications.

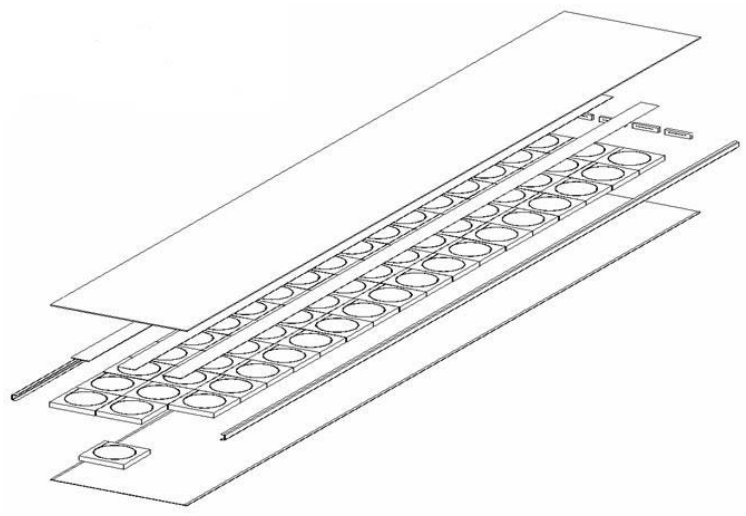

Fig. 3. Schematic drawing of one CPR upgrade module.

\section{TESTS AND DETECTOR PERFORMANCE}

I $\mathrm{N}$ order to optimize the final design of the detector, several tests were performed to compare different kinds of scintillators, fibers, and groove shapes. As the new detector is placed in the space previously occupied by the old one, the dimensions are fixed and the studies were mostly aimed at maximizing the light yield, in terms of the number of photoelectrons (pe) per MIP detected.

The new CPR detector was designed with the goal of being sensitive to the measurement of single particles, which requires a minimum signal of 5 pe/MIP. Unlike the old CPR, the new detector has no dead regions in $\eta$, thus allowing complete coverage. On the other hand, the CCR is only designed to cover the cracks and detect early particle showers. Preliminary tests indicated a light yield of 7 pe/MIP at the tile exit, which allows extension of the measurement of the electromagnetic shower energy to the crack regions.

Different tile/fiber configurations have been investigated and the results compared. Cosmic rays were used to test the tile response to MIPs. In order to increase the sampling of the light from the scintillator tile, a fiber is inserted into a groove whose path allows loading of multiple loops. Two different groove shapes have been compared: keyhole and square grooves. In order to provide good optical coupling between fiber and scintillator tile, optical cement was used in some tests.

In Figure 4, the light yield from a 2-loop spiral keyhole groove (solid shaded histogram and fit) compares well to the light yield from a fiber glued in a 4-loop square groove (dotted histogram). The keyhole-shaped groove allows capture of light from all sides while mantaining, at the same time, the fiber inserted in place without the use of glue, thus simplifying the assembly procedure. In order to maximize the light yield, the scintillator tiles are also polished on all sides and wrapped in an aluminized mylar foil, acting as a reflector and separator between adjacent tiles.

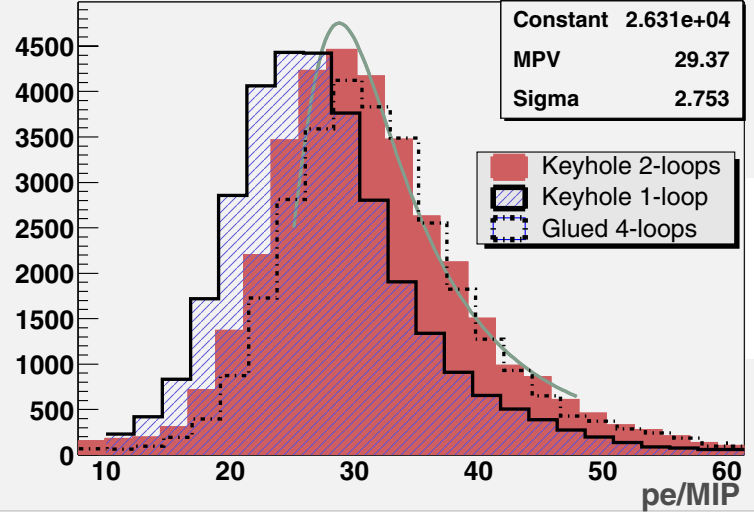

Fig. 4. Comparison of different methods of fiber/tile configurations. Light yield is optimized when the fiber is inserted in a 2-loop keyhole groove.

Owing to the small size of the scintillator tile, the light travels a relatively short distance to the WLS fibers. The light yield may be increased by using a scintillator with a high dopant concentration and a short attenuation length. WLS fibers manufactured by Kuraray [7] and PolHiTech [8] were compared, and the attenuation length was measured to be approximately $5 \mathrm{~m}$ for both. Kuraray fibers were chosen for their previous good performance in other CDF sub-detectors. The light yield from multiple fiber loops inserted into the groove reaches a plateau when the increase of light collection is compensated by the attenuation length of the fiber. The choice of two loops for the Kuraray WLS fibers is a compromise between assembly and light yield optimization. The new CPR reads out only one end of the fiber, but much of the light transmitted in the other direction is recovered $(\sim 30 \%)$ by mirroring that end of the fiber. The Kuraray multi-clad Y11 WLS fiber embedded in a 2-loop spiral keyhole groove yields $\sim 30$ pe/MIP at the tile exit and is the preferred choice for the final detector.

Two different scintillator tiles were used for comparison, "Dubna" [9] and Bicron 408 [10]. Light yields obtained were similar within 5\% (Fig. 5). Dubna tiles are prepared with polystyrene, a material with radiation hard properties, and were chosen in the final design.

The clear fibers that transport the light to the multi-channel Hamamatsu PMTs are manufactured by PolHiTech and their attenuation length was measured to be $\sim 7 \mathrm{~m}$, conforming to specifications (Fig. 6). More than $20 \mathrm{~km}$ were needed for the entire project.

PMT uniformity has been tested prior to installation and conformed to specifications from Hamamatsu. The channelto-channel variation has a maximum spread of 3:1; however, the fiber uses only the center of each pixel, and tests indicate a variation of only $\sim 10-15 \%$ among pixels. The cross talk between direct neighbors is $\sim 1-2 \%$. The high voltage supplied to the 192 PMTs is controlled by a CAEN [11] crate (SY527) with eight 24-channel 932AN distributor boards. Voltages have been adjusted to obtain approximately the same gain among PMTs. Calibration constants have been included in a prelimi- 


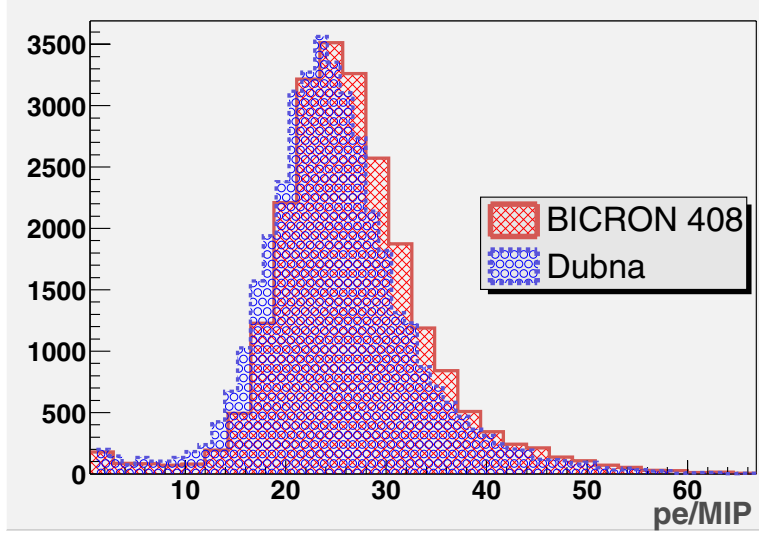

Fig. 5. Light yield for two different scintillator tiles. In this test, a nonmirrored WLS fiber by PolHiTech with a 4-loop configuration was used. Light yields from Bicron 408 and Dubna tiles are within $5 \%$.

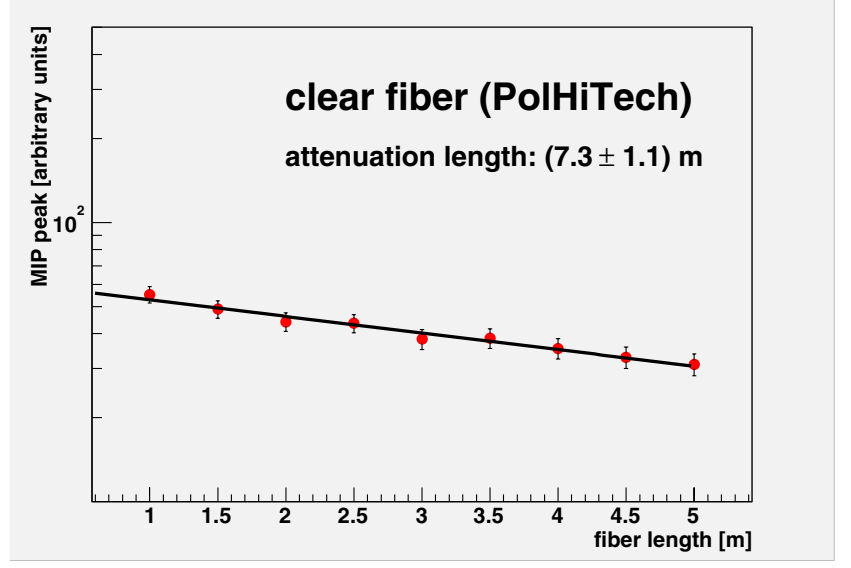

Fig. 6. Relative light yield response measured as a function of the length of one PolHiTech production clear fiber.

nary database to account for variation of the response among different channels and will be further adjusted with colliding beam data, once the Tevatron resumes collisions.

\section{Detector ASSEMbly}

$\mathbf{I}$ $\mathrm{N}$ preparation for detector assembly, the WLS fibers spliced to clear fibers and then glued into plastic connectors were grouped in four bunches per module. The light transmission after splicing was measured to be $\sim 92-93 \%$, with good reproducibility and small uncertainties. Prior to module assembly, both fibers and tiles were individually tested for light yield uniformity using a radioactive source and visually inspected for damage. During module assembly (Fig. 7), each tile was first positioned in its location and the fiber then inserted in the keyhole groove and fixed in place, away from the tiles' borders. The same procedure was repeated for all tiles, moving away from the connectors, until completion of the module. The fibers of the six farthest tiles were glued with optical cement to increase the light yield and compensate for a longer fiber path. In order to ensure good quality control of module production, fibers were again individually checked for damage after assembly. After closing the aluminum shell with lighttight sealant, each module was then scanned with a radioactive source and the light yield measured for all tiles. In Figure 8, the relative light yield response for the tiles used in the production modules has a spread of $\sim 18 \%$.

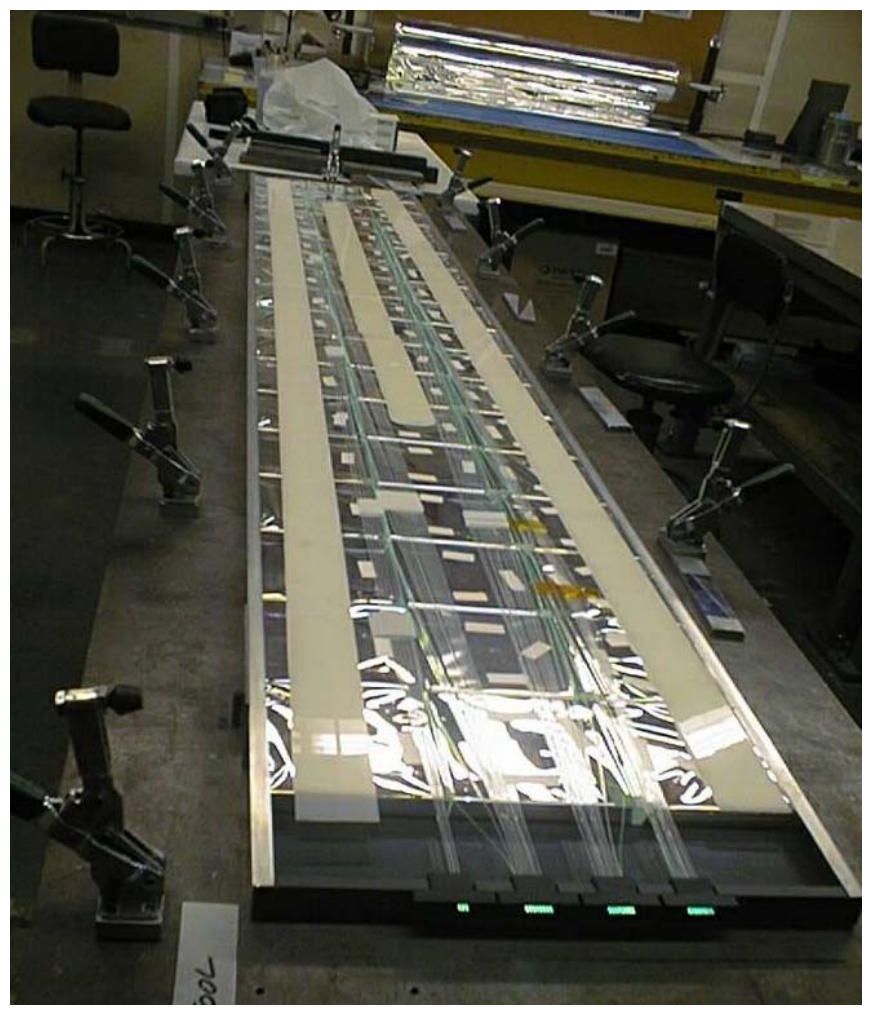

Fig. 7. Assembly of one CPR module: All 54 tiles are wrapped in aluminized mylar foil and the fibers routed to the four output connectors.

After installation, constant monitoring of detector response was performed using cosmic rays. The response of all installed modules was measured (Fig. 9) and preliminary estimates indicate a light yield of 12 pe/MIP after the whole optical chain (when the measurement is averaged over all the CPR modules), well above the design specifications of 5 pe/MIP.

\section{CONCLUSIONS}

$\mathbf{T}$ HE central preshower detector for the CDF experiment was designed and built after careful consideration of the physics issues that need to be addressed in the next few years at the Tevatron. Particular attention was devoted to design studies aimed at optimizing the light yield and reliability. Detector production was completed smoothly and quality control was performed at various stages before, during, and after the construction phase. Detector installation took place during the fall 2004 in a timely fashion. Tests indicate that the final detector meets and exceeds design requirements. Indeed, preliminary measurements of light yield show a two-fold increase with respect to design goals. The calibration performed prior to installation allows valuable monitoring of detector performance. 


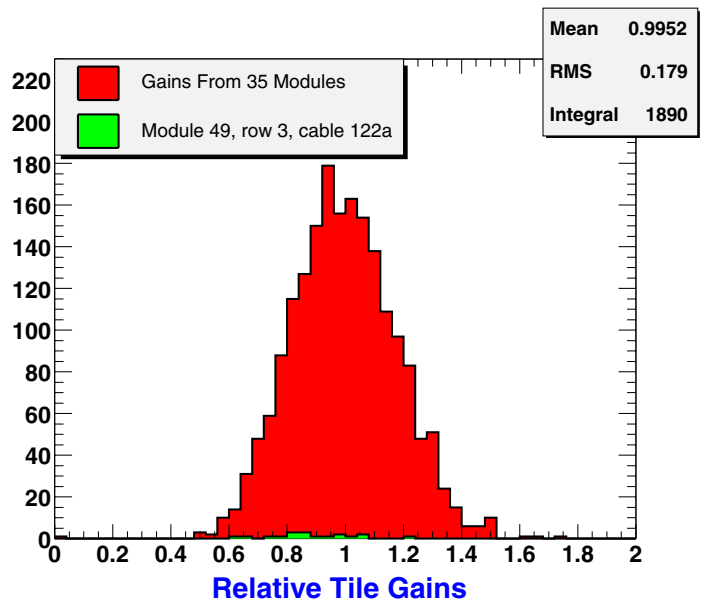

Fig. 8. Relative light yield response of the production scintillator tiles.

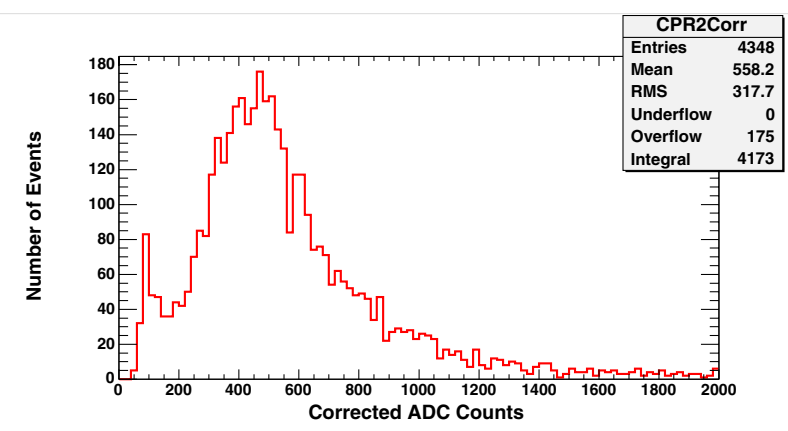

Fig. 9. ADC count distribution from cosmic ray data for the CPR modules after detector installation at CDF. A light yield of $12 \mathrm{pe} / \mathrm{MIP}$ is estimated after the whole optical path.

\section{ACKNOWLEDGMENTS}

A ANY thanks to the Argonne, INFN and Fermilab staffs for their vital contribution to the production, assembly, and installation of the detector.

\section{REFERENCES}

[1] F. Abe et al. (CDF Collaboration), "Evidence for top quark production in $\bar{p} p$ collisions at $\sqrt{s}=1.8 \mathrm{TeV}$ ", Phys. Rev. D 50 (1994) 2966.

[2] S. Lami et al., Proc. of IX Int. Conf. on Calorimetry in High Energy Physics (Calor 2000), 555

[3] F. Abe et al. (CDF Collaboration), "Searches for new physics in diphoton events in $p \bar{p}$ collisions at $\sqrt{s}=1.8 \mathrm{TeV}$ ", Phys. Rev. D 59 (1999) 092002.

[4] Another upgrade project for the CDF calorimeter includes the addition of timing information to both central and plug electromagnetic calorimeters, and is needed to filter out cosmic ray and beam related backgrounds. This project is described in more detail in Ref. [5].

[5] S. Kuhlmann et al., "The CDF Calorimeter Upgrade for Run IIb", Nucl. Instrum. Meth. A 518 (2004) 39.

[6] Each module of the old CPR gas detector had a structure of 16 strips, running longitudinally across five calorimeter towers and segmented in the azimuthal angle $\phi$. Each calorimeter wedge was covered by two modules, thus creating a dead region between the two.

[7] Kuraray America Inc., New York, NY 10166, USA.

[8] PolHiTech SRL, 67061 Carsoli (AQ), Italy (www.polhitech.it).

[9] Provided by the Dubna laboratory.

[10] Saint-Gobain Crystals \& Detectors, USA (www.bicron.com).

[11] CAEN, 55049 Viareggio (LU), Italy (www.caen.it). 